Real-time measurement of the three-axis contact force distribution using a flexible capacitive polymer tactile sensor

This article has been downloaded from IOPscience. Please scroll down to see the full text article.

2011 J. Micromech. Microeng. 21035010

(http://iopscience.iop.org/0960-1317/21/3/035010)

View the table of contents for this issue, or go to the journal homepage for more

Download details:

IP Address: 141.211.173.82

The article was downloaded on 06/04/2012 at 16:55

Please note that terms and conditions apply. 


\title{
Real-time measurement of the three-axis contact force distribution using a flexible capacitive polymer tactile sensor
}

\author{
Hyung-Kew Lee, Jaehoon Chung, Sun-Il Chang and Euisik Yoon ${ }^{1}$ \\ Department of Electrical Engineering and Computer Science University of Michigan 1301 Beal Ave, \\ Ann Arbor, MI 48109, USA \\ E-mail: esyoon@umn.edu
}

Received 17 August 2010, in final form 22 December 2010

Published 8 February 2011

Online at stacks.iop.org/JMM/21/035010

\begin{abstract}
In this paper, we report real-time measurement results of various contact forces exerted on a new flexible capacitive three-axis tactile sensor array based on polydimethylsiloxane (PDMS). A unit sensor consists of two thick PDMS layers with embedded copper electrodes, a spacer layer, an insulation layer and a bump layer. There are four capacitors in a unit sensor to decompose a contact force into its normal and shear components. They are separated by a wall-type spacer to improve the mechanical response time. Four capacitors are arranged in a square form. The whole sensor is an $8 \times 8$ array of unit sensors and each unit sensor responds to forces in all three axes. Measurement results show that the full-scale range of detectable force is around $0-20 \mathrm{mN}(250 \mathrm{kPa})$ for all three axes. The estimated sensitivities of a unit sensor with the current setup are 1.3, 1.2 and $1.2 \% / \mathrm{mN}$ for the $x$-, $y$ - and $z$-axes, respectively. A simple mechanical model has been established to calculate each axial force component from the measured capacitance value. Normal and shear force distribution images are captured from the fabricated sensor using a real-time measurement system. The mechanical response time of a unit sensor has been estimated to be less than $160 \mathrm{~ms}$. The flexibility of the sensor has also been demonstrated by operating the sensor on a curved surface of $4 \mathrm{~mm}$ radius of curvature.
\end{abstract}

(Some figures in this article are in colour only in the electronic version)

\section{Introduction}

Tactile sensors are gaining more attention than before in robot and medical research fields. The advancement of robot technology enabled the implementation of humanoids such as ASIMO and HUBO in the early 21st century [1, 2]. As robot technology evolves, the significance of a tactile sensor is getting more recognized by the researchers who are trying to make robots conduct practical tasks such as grasping and moving objects. Humans are able to manipulate objects dexterously and conduct a variety of tasks with their hands. One of the critical factors that make it possible is the existence of a sophisticated tactile sensor system in human beings. Therefore, in order for the robots to conduct practical tasks that humans do without difficulty, they have

\footnotetext{
1 Author to whom any correspondence should be addressed.
}

to be equipped with tactile sensors which have similar form factor and functionalities as humans. This was the original motivation for tactile sensor research. Tactile sensors are also becoming one of the key components in medical devices and tools. One of the most impressive recent advancements in medical device research is artificial arms for amputees. A few research groups demonstrated brain-controlled artificial arms that the recipients can control as they did before they lost their real arms, though not perfect yet [3]. These artificial hands have primitive tactile sensors. There is still plenty of room for improvement. Besides the application to anthropomorphic hands, tactile sensors are being actively researched for other medical devices. There is a commercial medical device which diagnoses breast cancer by using a high sensitive tactile sensor [4]. Minimally invasive surgery (MIS) is also adopting tactile sensors. MIS is one of the fastest developing surgery techniques because it minimizes the post-surgery stress and 
pain of patients. Since the tools used in MIS are smaller than human hands, the primary focus of the research is the development of small and accurate tactile sensors that can be integrated in small surgery tools. Many research groups are developing tactile sensors targeting MIS applications using various technologies [5]. Among these various devices, in this paper, we focused on the tactile sensors for anthropomorphic hands applied to humanoids and artificial prosthetic hands.

It is desirable for the tactile sensor for such applications to have similar characteristics to human skin. Therefore, the requirements for the tactile sensor for such applications are flexibility, compliance, robustness, around $1 \mathrm{~mm}$ spatial resolution for finger, and potential for large area deployment $[6,7]$. Most of all, the tactile sensor for anthropomorphic hands should be able to detect both normal and shear force in order to maneuver objects freely [8]. For years, diverse types of tactile sensors have been developed for the three-axis measurement of the applied contact force. Some of the works focused on the slip detection of an object using PVDF and relatively simple structure $[9,10]$. This type of sensor is not able to measure the static shear force because PVDF responds only to the change of the contact force. Many research groups have adopted MEMS technology to implement the three-axis sensors because it is relatively easy to fabricate accurate threeaxis sensors of sub-millimeter size [7, 11, 12]. Such MEMS technology is indispensable to realize tactile sensors with a fine spatial resolution and sensitivity comparable to human fingers. One report demonstrated a decent result of shear force and slip detection by using a silicon-based three-axis sensor element packaged in polyurethane [7]. Array sensors are also realized with silicon MEMS technology [11, 12]. However, they are not flexible because they have a rigid silicon substrate. They also have size limit due to the cost of the silicon substrate and MEMS processes. In order to make the array sensor flexible, a few three-axis tactile sensors have been implemented on a flexible polymer substrate such as polyimide [13-15]. They used piezoresistors in the form of strain gauge to sense the deflection of a micromachined sensing membrane. Although polyimide is flexible enough to bend, it is not as compliant as human skin. And its size is still limited.

In order to meet the requirements on tactile sensors for anthropomorphic hands, we took a different approach in our previous works $[6,16]$. First, we introduced an elastomer material, polydimethylsiloxane (PDMS), as a base structural material of the sensor. We developed a novel fabrication technology to realize a compliant capacitive tactile sensor using PDMS whose mechanical characteristics are relatively close to that of human skin. Second, we implemented an expandable sensor structure [6]. Our recent work has successfully demonstrated the feasibility of a three-axis sensor and captured images of the contact force distribution in both normal and shear directions [16]. However, we could capture only the static pressure distribution from a $4 \times 4$ live array out of $8 \times 8$ because the sensor structure had a mechanical problem. In this paper, we report an improved sensor design and the real-time measurement results of the contact force distribution.

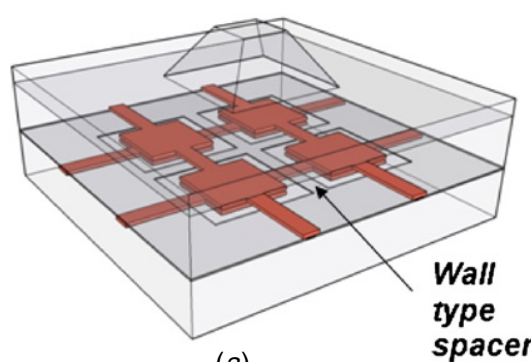

(a)

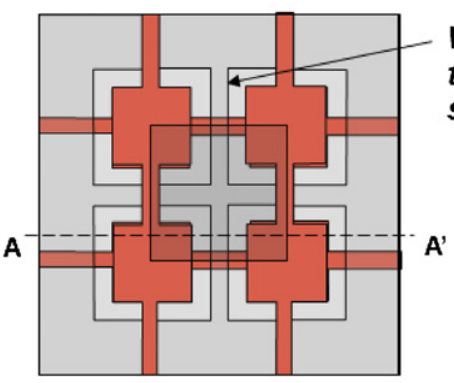

(b)

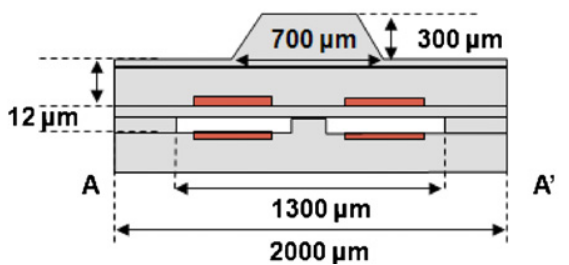

(c)

Figure 1. Conceptual diagram of a unit sensor of the proposed three-axis capacitive tactile sensor: $(a)$ schematic view, $(b)$ plane view, and $(c)$ cross-sectional view along $\mathrm{AA}^{\prime}$.

\section{Sensor structure and operation}

The problem in the previous structure was that there was too large an exposed PDMS area around the pillar, resulting in many dead unit sensors with the facing PDMS stuck together during the fabrication process. Besides, even in live unit sensors, the facing PDMS areas contact each other when high pressure is applied, making their recovery slow. It took more than a second for the previous structure to recover fully once the two faces made a contact due to high pressure. Therefore, we replaced the pillar structure with a wall-type spacer between the four capacitors in order to reduce the facing PDMS area and increase the recovery force of the unit sensor in this work.

Figure 1(a) shows the revised unit sensor structure. It consists of five PDMS layers like its predecessor: a bump layer, an upper electrode layer, an insulation layer, a spacer layer and a lower electrode layer. The four capacitors in a sensor are separated by a wall-type spacer. The rest of the structure is the same as the previous three-axis sensor. Figure 1(b) shows the top view and figure 1(c) the cross-sectional view along the $\mathrm{AA}^{\prime}$ line in figure $1(b)$ with dimensions of features. The air gap between the top and bottom electrodes is $6 \mu \mathrm{m}$. The thickness of the insulation layer is $6 \mu \mathrm{m}$. Each capacitor has the area of $400 \times 400 \mu \mathrm{m}^{2}$. The width of the wall-type spacer among capacitors is $100 \mu \mathrm{m}$. The bump has $300 \mu \mathrm{m}$ height to enhance the sensitivity of 


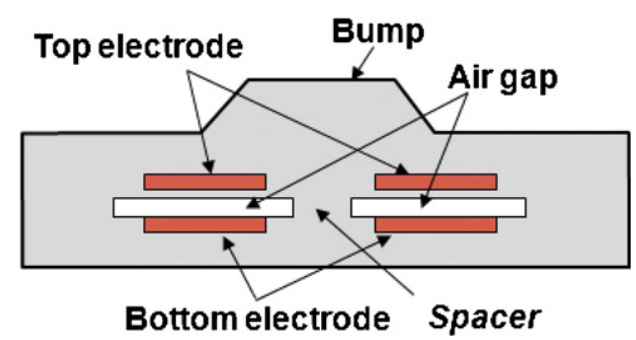

(a)

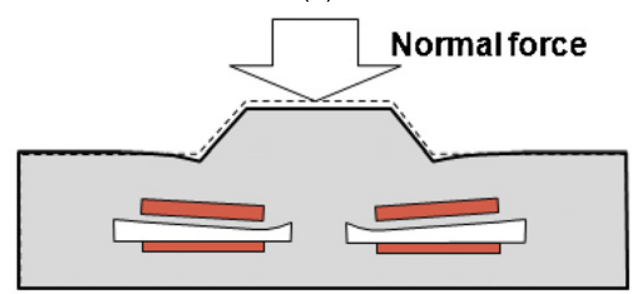

(b)

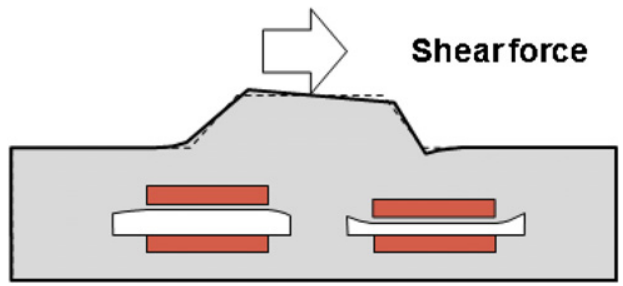

(c)

Figure 2. Operation principle of the three-axis tactile sensor: (a) cross-sectional view of a tactile cell without applied forces, (b) response to normal force, and (c) response to shear force.

differential capacitance change when shear stress is applied. It is placed at the center of a cell and its four corners sit at the center of each capacitor. The principle of operation is the same as in the previous pillar structure. Figure 2 represents the deformation of the unit sensor under normal and shear contact pressures. The bump and the wall structure play a critical role for proper operation. When a normal force is applied on top of the bump, the upper PDMS layer is compressed and the air gap is reduced as shown in figure $2(b)$. All four capacitors show symmetric deformation around the wall. When a shear force is applied on top of the bump, the bump deforms as shown in figure $2(c)$ and the force generates a torque in the upper electrode layers around the wall. As a result, the air gap on the left side reduces, while that on the right side increases. The height of the bump should be large enough to generate a sufficient torque.

This basic operation principle was verified in our previous report through mechanical simulation [16]. According to the simulation results, the mechanical deformation under the normal and shear stress on the bump is close to the deformation described in figure 2 and linearly proportional to the applied stress in both normal and shear directions. The results also show independent response in the normal and shear directions, which enables easy measurement of each axial component. The crosstalk between unit sensors was estimated to be negligible.

\section{Fabrication}

Unlike the previous fabrication process which has a polymer mold process for the pillar, in this work, the polymer mold process was removed since the wall-type spacer is a part of the spacer layer-another improvement for better process yield. Because we use PDMS as a structural material, it is important to precisely control the thickness and uniformity of PDMS layers. Therefore, we developed PDMS (Sylgard 184 $\mathrm{A}: \mathrm{B}=10: 1$ in weight) spin-coating and planarization process in our previous report. We could control the thickness of vulcanized PDMS from 21 to $300 \mu \mathrm{m}$ by spin-coating within $\pm 7 \%$ variation. We also could achieve $\pm 3.5 \%$ uniformity for $300 \mu \mathrm{m}$ PDMS over a 4 inch wafer by using our customized planarization stage [6].

The fabrication process of each layer is explained in figures $3(a)-(c)$. Each layer is processed separately and bonded together after oxygen plasma treatment. For the electrode layers (figure 3(a)), LOR 20B from Microchem is spin-coated about $10 \mu \mathrm{m}$ on a silicon wafer. LOR works

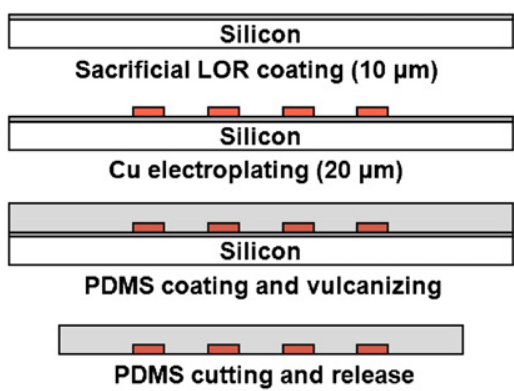

(a)

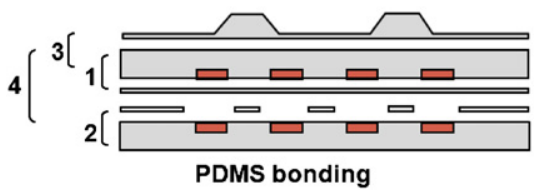

(d)

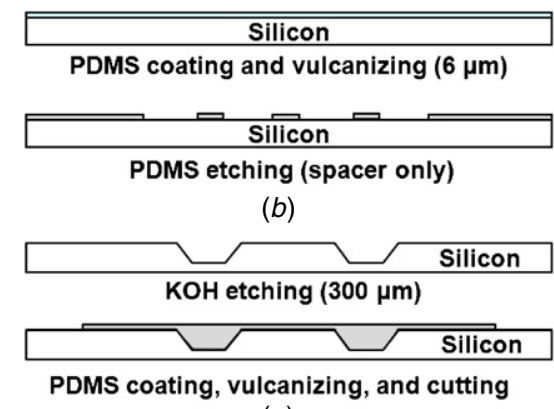

(c)

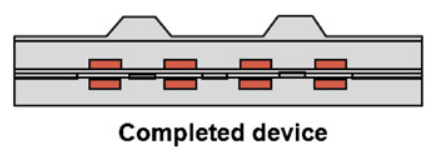

(e)

Figure 3. Fabrication process of the proposed three-axis tactile sensor: $(a)$ the electrode layer, $(b)$ the insulation layer and the spacer layer, $(c)$ the bump layer, $(d)$ the bonding process, and $(e)$ the completed device. 
as a sacrificial layer. Then, a copper electrode $(20 \mu \mathrm{m})$ is electroplated exploiting AZ9260 photoresist as a plating mold. Next, titanium (200 $⿱$ $)$ is sputtered as an adhesion layer. After $\mathrm{O}_{2}$ plasma treatment of the titanium surface for $8 \mathrm{~min}$ at $50 \mathrm{~W}$, PDMS is spin-coated about $300 \mu \mathrm{m}$ and cured on the planarization stage. PDMS should be cured at room temperature to prevent the layers from being deformed after release due to the thermal expansion difference between the copper and PDMS layers. After vulcanizing PDMS at room temperature, the electrode layer is cut and peeled off. We tested three different methods of releasing the finished electrode layer. The first method is dissolving the sacrificial LOR in the 4:1 AZ400K developer. This method takes several hours. The second method is immersing the substrate in acetone and applying an ultrasonic wave. The acetone and ultrasonic waves physically damage the sacrificial LOR and release the electrode layer in $10 \mathrm{~min}$. However, we observed permanent shrinkage of PDMS. The third method is cleaving the wafer in the middle of the electrode layer and peeling it off. We have used the third method in this work since it releases the finished electrode layer quickly leaving it unaffected by the process.

The insulation and spacer layers (figure $3(b)$ ) are coated on silicon wafers by spin-coating PDMS diluted with n-hexane (Sylgard 184 A:B:n-hexane = 10:1:10 in weight). The spin speed and time are $5000 \mathrm{rpm}$ and $30 \mathrm{~s}$, respectively. Then, they are cured in a convection oven at $90{ }^{\circ} \mathrm{C}$ for $90 \mathrm{~min}$. The thickness of the cured PDMS is $6 \mu \mathrm{m}$. A $100 \mathrm{~nm}$ platinum layer is sputtered on the substrate before applying PDMS to weaken PDMS adhesion to the substrate for detachment which will be performed later. The spin-coated PDMS is patterned and etched using reactive ion etching (RIE) for $45 \mathrm{~min}$ with 3:1 $\mathrm{SF}_{6} / \mathrm{O}_{2}$ gas at $100 \mathrm{mTorr}$ to form a spacer layer with wall structures. The AZ4330 photoresist of thickness $8 \mu \mathrm{m}$ was used to pattern the spacer layer. The wet process for a PDMS layer requires great caution because the adhesion of PDMS to platinum and photoresist is weak. The photoresist is developed with slight agitation of the developer (4:1 AZ400K), and the rinse should be done in a slow flow of DI water. The hardened photoresist, after plasma etching, is stripped by the developer after flood exposure to UV. The etch rate of PDMS and the selectivity to the photoresist are $150 \mathrm{~nm} \mathrm{~min}^{-1}$ and $1.5: 1$, respectively.

As for the bump layer (figure 3(c)), a silicon wafer is etched in $\mathrm{KOH}\left(80^{\circ} \mathrm{C}, 4 \mathrm{~h}\right.$ and $\left.20 \mathrm{~min}\right)$ to form a mold for the bump patterns. Thermal oxide of $1 \mu \mathrm{m}$ thickness is used as a mask layer. Then, platinum is deposited and the PDMS is coated by $100 \mu \mathrm{m}$ and vulcanized. PDMS fills the $300 \mu \mathrm{m}$ bump molds pretty quickly and uniformly.

Finally, the fabricated five layers are aligned and bonded together using a conventional contact aligner (Karl Suss MJB3) with slight modification according to the sequence shown in figure $3(d)$. We used a transparent film as a carrier for the fabricated PDMS layers during cleaning, $\mathrm{O}_{2}$ plasma treatment and bonding, for it gives adequate stiffness for support and flexibility for peel-off of the PDMS layers after bonding. Figure 3(e) displays the completed device.

The photographs in figure 4 are the fabricated $8 \times 8$ three-axis tactile sensors $(16 \times 16$ capacitors $)$. The size of the
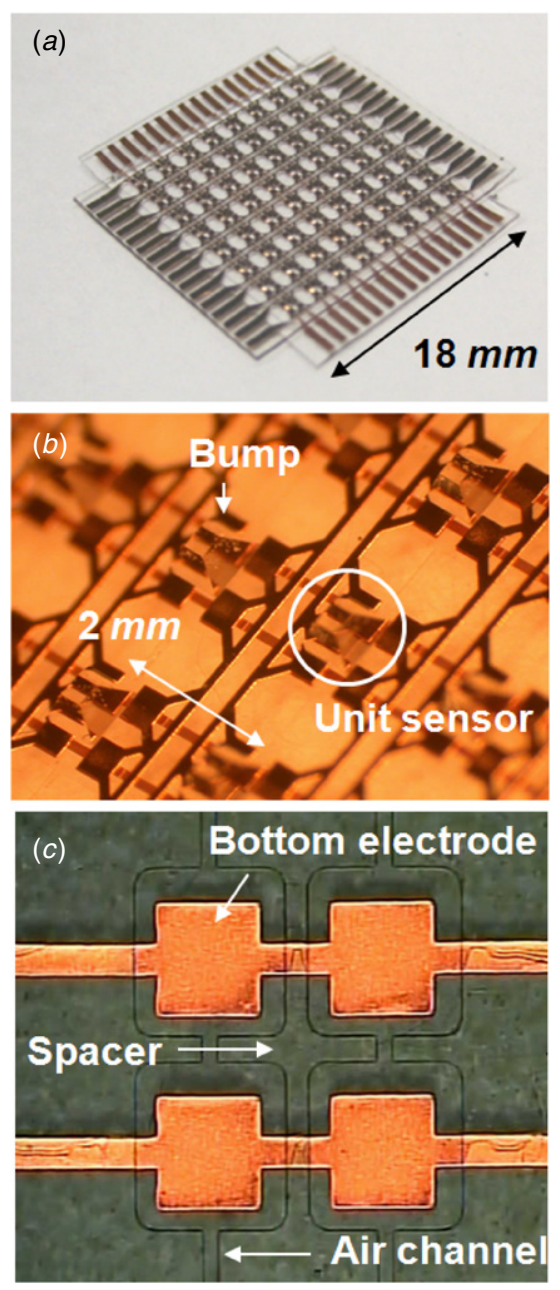

Figure 4. (a) Photograph of the completed three-axis tactile sensor (an $8 \times 8$ array of unit sensors), $(b)$ the magnified view of the unit sensor array, and $(c)$ the magnified view of a unit sensor showing the details of bottom electrodes and the wall spacer which separates four capacitors.

sensor area is $18 \times 18 \mathrm{~mm}^{2}$ excluding interconnection lines. The pitch between unit sensors is $2 \mathrm{~mm}$. Four capacitors are separated by the wall-type spacer as shown in figure 4(c).

\section{Measurements and discussions}

\subsection{Unit sensor measurement}

A force gauge with $1 \mathrm{mN}$ resolution and a motorized precision translation stage of about a $100 \mathrm{~nm}$ resolution have been used for unit sensor characterization as in the previous work [16]. Normal and shear forces were applied to the bump with two types of probes: a straight bar for the normal force and an ' $L$ ' shaped bar for shear forces. They were installed on the force gauge and actuated by the motorized stage. The capacitance was measured and recorded using a custom circuit board and National Instruments' data acquisition pad. The initial capacitances (C11, C12, C21 and C22) were measured as 203, 204, 190 and $205 \mathrm{fF}$, respectively. An average value from 50 measurements was taken for every data point. The theoretical initial capacitance value is $172 \mathrm{fF}$. The discrepancy might be due to the fabrication process variation. 


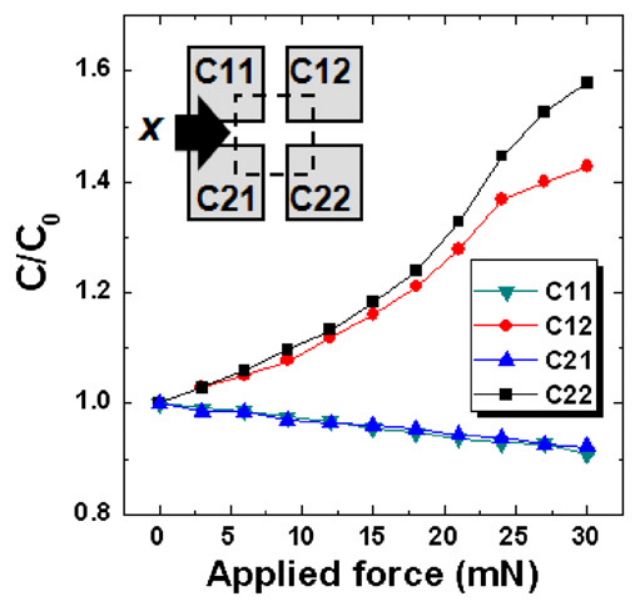

(a)

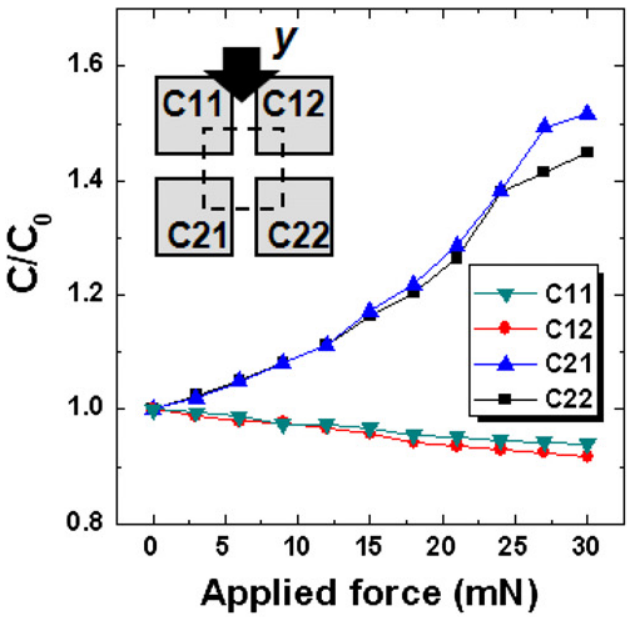

(B)

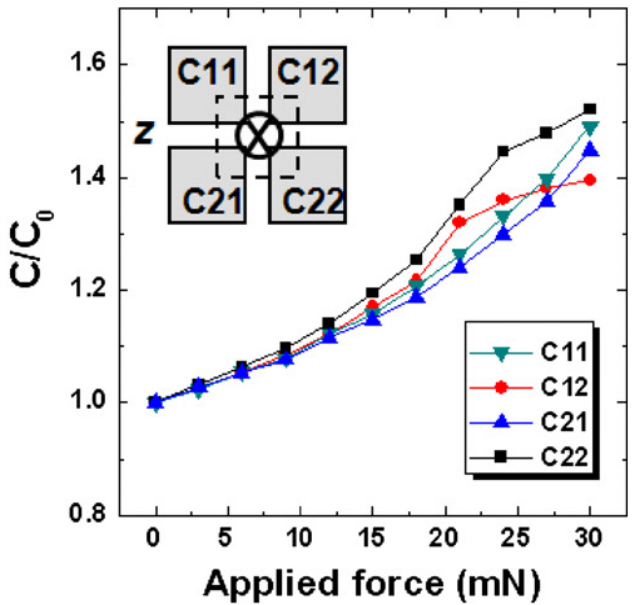

(c)

Figure 5. Measured capacitance values of a unit sensor as a function of applied forces in three axes: $(a) x,(b) y$, and $(c) z$. The capacitance is normalized by its initial value.

We exerted axial forces in each direction to the bump and plotted the normalized capacitance value in figure 5 . The normalized capacitance values from all four capacitors exhibited the same behavior for the pure $z$-directional force as expected, though not perfectly the same. The abrupt change in the slope of $\mathrm{C} 12$ and $\mathrm{C} 22$ in figure 5(c) implies that the gap begins to be closed. Technically, all four capacitors should

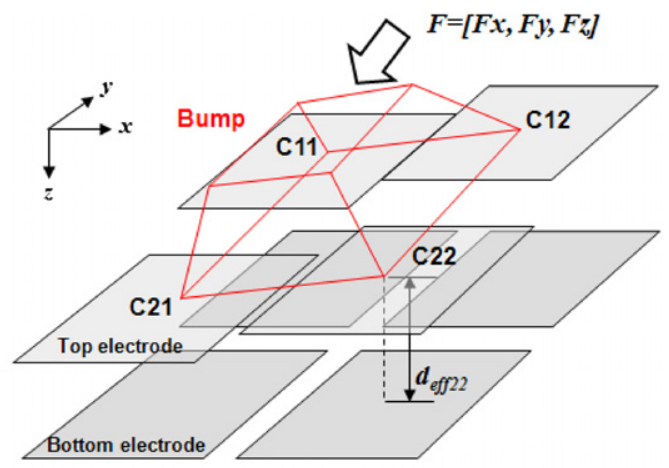

(a)

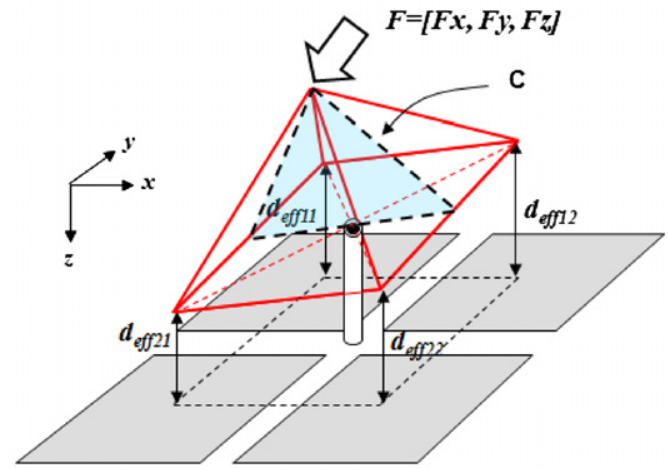

(b)

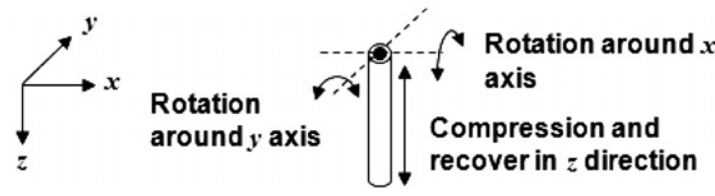

(c)

Figure 6. A simplified mechanical model of a unit sensor to calculate the force component in each axis: $(a)$ movement of top electrodes of four capacitors and a bump when a force $F$ is applied to the bump, $(b)$ a simplified model of a unit sensor consisting of a rigid pyramid-shaped bump, and (c) a mechanical spring element with three degrees of freedom (DOF). ' $C$ ' is a cross-section of the pyramid model.

have exactly the same response. However, there are always variations and errors in the fabrication process. Additionally, the probe tip is not perfectly parallel to the electrodes during experiments. The air gap is only $6 \mu \mathrm{m}$, which is very small compared to the size of the unit sensor $(1300 \mu \mathrm{m})$. It is extremely difficult to make the tip surface and the four electrodes parallel with sub-micron accuracy in such a wide area without any visual feedback in our measurement setup. In figure 5(a), the capacitance value from $\mathrm{C} 12$ and $\mathrm{C} 22$ increased while the others decreased as we described in section 2 for a pure $x$-force. Figure 5(b) shows the same behavior for a shear force in the $y$-direction. One thing that should be noted is the asymmetric response around the zero between the two sets of capacitors (for instance, C11, C12 and C21, C22 in figure $5(b)$ ) from shear stress. We verified the cause of this asymmetry in the previous work [16]. The soft PDMS bump deforms severely as the tip pushes it from the side. This deformation allows the tip to penetrate into the bump so as to limit the movement of the PDMS under the tip, which works as an additional normal force. 


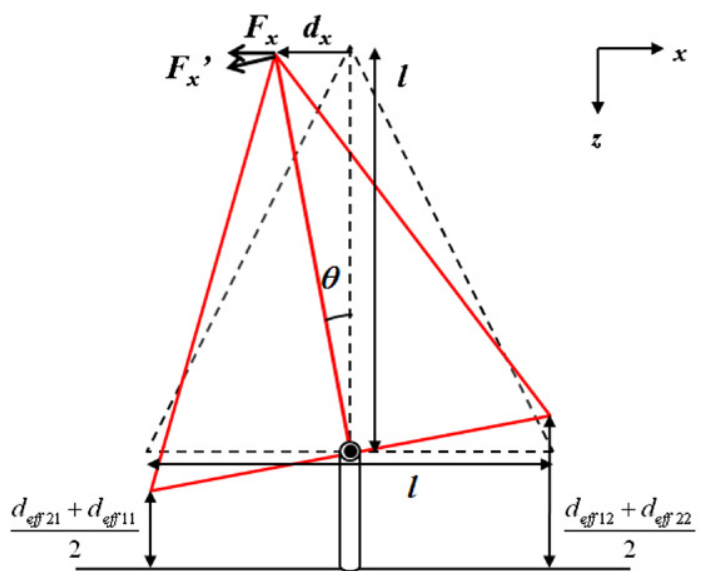

(a)

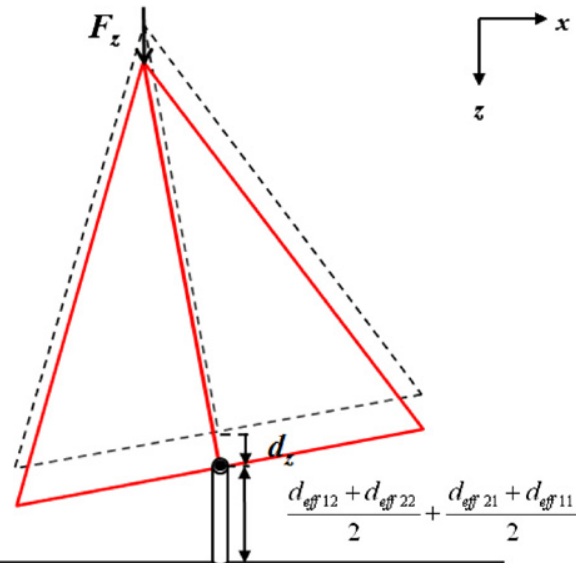

(b)

Figure 7. (a) The movement of the cross-section of the pyramid model shown in figure 6 under a shear force in the $x$-direction. $(b)$ The movement of the cross-section of the pyramid model under an additional normal force in the $z$-direction.

In order to calculate three axial components of input forces, a mechanical model has been established and is illustrated in figure 6. Figure 6(a) gives a simplified mechanical description of a unit sensor under a force $F$. The effective gap distance (EGD, $d_{\text {eff }}$ ) is calculated first, assuming that the top electrode in a capacitor moves parallel to the bottom electrode. Figure $6(b)$ illustrates the mechanical model of the unit sensor. It consists of a rigid bump of a pyramid shape along with a special spring element shown in figure 6(c). The spring element has three degrees of freedom (DOF) as shown in the figure. The spring element allows rotational movement around the $x$ - and $y$-axes and translation movement along the $z$-axis only.

Figure 7(a) shows the movement of the cross-section $C$ in figure $6(b)$ under $F_{x}$. The torque exerted on the bump by $F_{x}$ is given as

$$
\tau_{x}=\kappa_{x} \theta=F_{x}^{\prime} \times l=F_{x} \times \cos \theta \times l
$$

where $\kappa_{x}$ is the torsion coefficient in the $x$-direction. However, while $l$, the height of the bump and the gap between centers of each capacitor, is $700 \mu \mathrm{m}$, the maximum EGD is limited to only $6 \mu \mathrm{m}$ which is the thickness of the air gap between

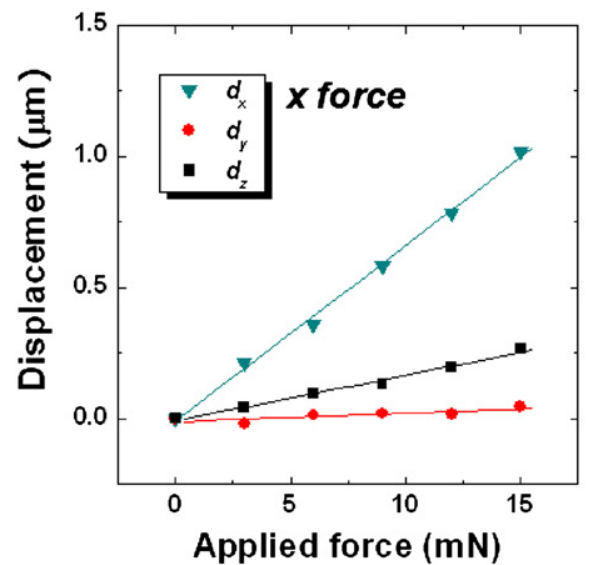

(a)

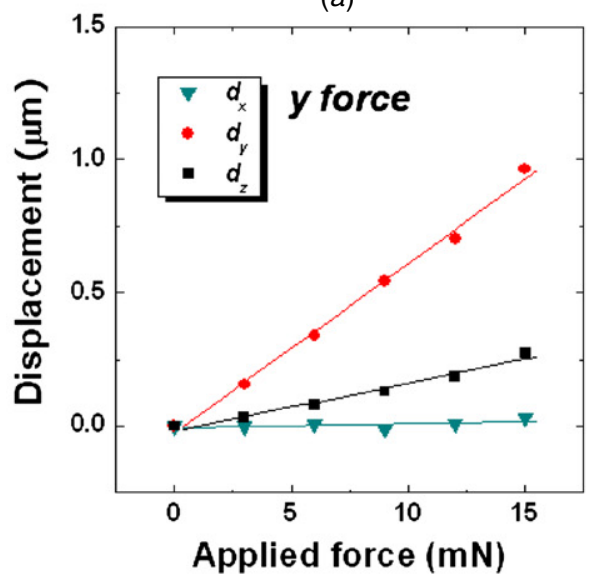

(b)

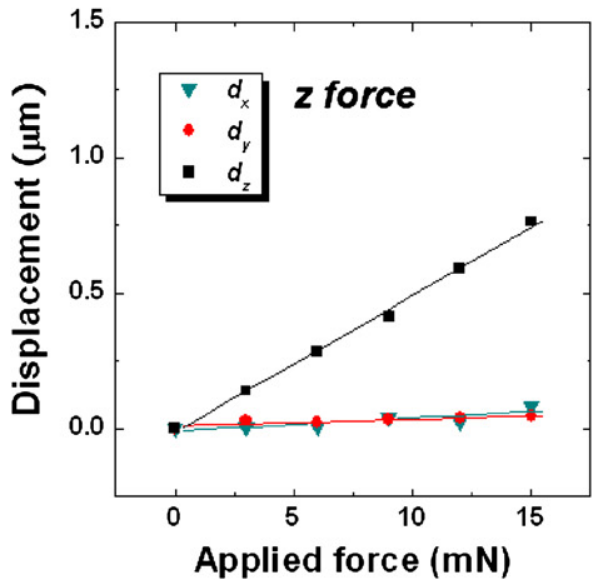

(c)

Figure 8. The decomposed three axial displacements for $(a) x,(b) y$ and $(c) z$ axial forces from 0 to $15 \mathrm{mN}$.

electrodes. Therefore, the torque and the angular displacement can be easily approximated by

$$
\tau_{x} \cong F_{x} \times l
$$

and

$$
\theta \cong \frac{d_{x}}{l}
$$

respectively. Since we assumed the pyramid bump as a rigid body, $d_{x}$ can be calculated from EGDs as

$$
d_{x}=\left(\frac{d_{\mathrm{eff} 12}+d_{\mathrm{eff} 22}}{2}-\frac{d_{\mathrm{eff} 21}+d_{\mathrm{eff} 11}}{2}\right) .
$$




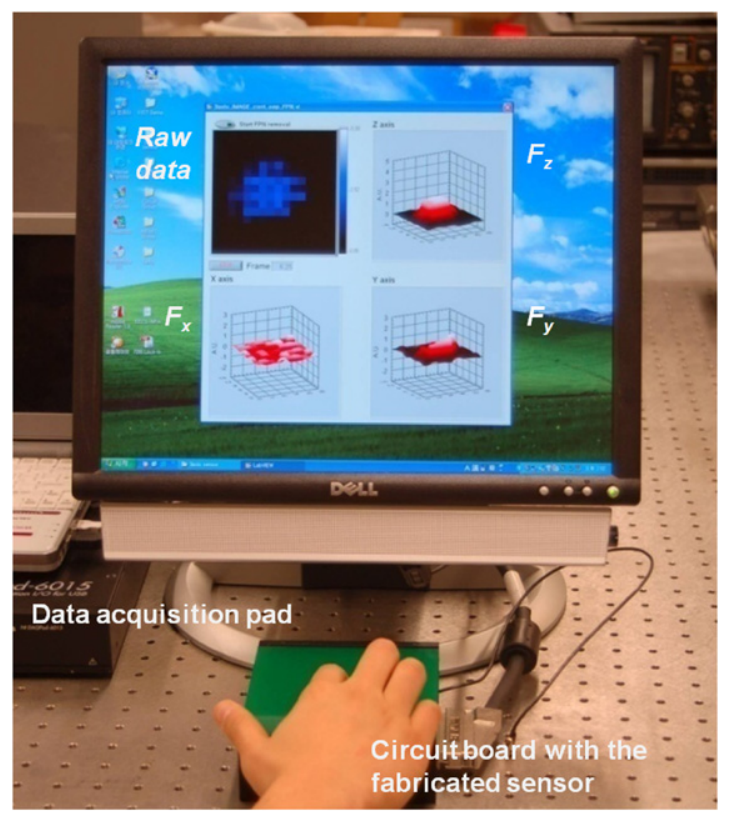

Figure 9. Experimental setup to monitor the three-axis distribution of the applied force in real time.

From equations (1)-(4), the relation between $F_{x}$ and EGDs from four capacitors can be summarized by

$$
\begin{aligned}
F_{x} & \cong \frac{\kappa \theta}{l} \cong \frac{\kappa_{x}}{l^{2}} \times d_{x} \\
& =\frac{\kappa_{x}}{l^{2}}\left[\frac{\left(d_{\mathrm{eff} 12}+d_{\mathrm{eff} 22}\right)-\left(d_{\mathrm{eff} 21}+d_{\mathrm{eff} 11}\right)}{2}\right] .
\end{aligned}
$$

$F_{y}$ can be described with the same logic as

$$
F_{y} \cong \frac{\kappa \theta}{l} \cong \frac{\kappa_{y}}{l^{2}}\left[\frac{\left(d_{\mathrm{eff} 12}+d_{\mathrm{eff} 11}\right)-\left(d_{\mathrm{eff21}}+d_{\mathrm{eff2}}\right)}{2}\right] .
$$

Equations (5) and (6) are in the form of Hooke's law in which spring constants are $\frac{k_{x}}{l^{2}}$ and $\frac{k_{y}}{l^{2}}$, respectively. Therefore, they can be considered as spring constants for shear forces in our model. Another thing we considered was the difference among initial EGDs from four capacitors due to the process error. They cause an offset from the calculated shear force. We defined a relative effective gap distance (REGD) as the difference between the current EGD and its initial value as $\Delta d_{\text {eff }}=d_{\text {effo }}-d_{\text {eff }}$ in order to compensate the offset and modify equations (5) and (6). The final equations of the shear force equation for the $x$ - and $y$-axes derived from our model are

$F_{x}=k_{x} d_{x}=k_{x}\left[\frac{\left(\Delta d_{\mathrm{eff} 12}+\Delta d_{\mathrm{eff22}}\right)-\left(\Delta d_{\mathrm{eff21}}+\Delta d_{\mathrm{eff} 11}\right)}{2}\right]$

and

$F_{y}=k_{y} d_{y}=k_{y}\left[\frac{\left(\Delta d_{\mathrm{eff} 12}+\Delta d_{\mathrm{eff} 11}\right)-\left(\Delta d_{\mathrm{eff} 21}+\Delta d_{\mathrm{eff} 22}\right)}{2}\right]$,

respectively.

Figure 7(b) describes the movement of the pyramid bump under $F_{z}$. Because the movement of the bump is restricted
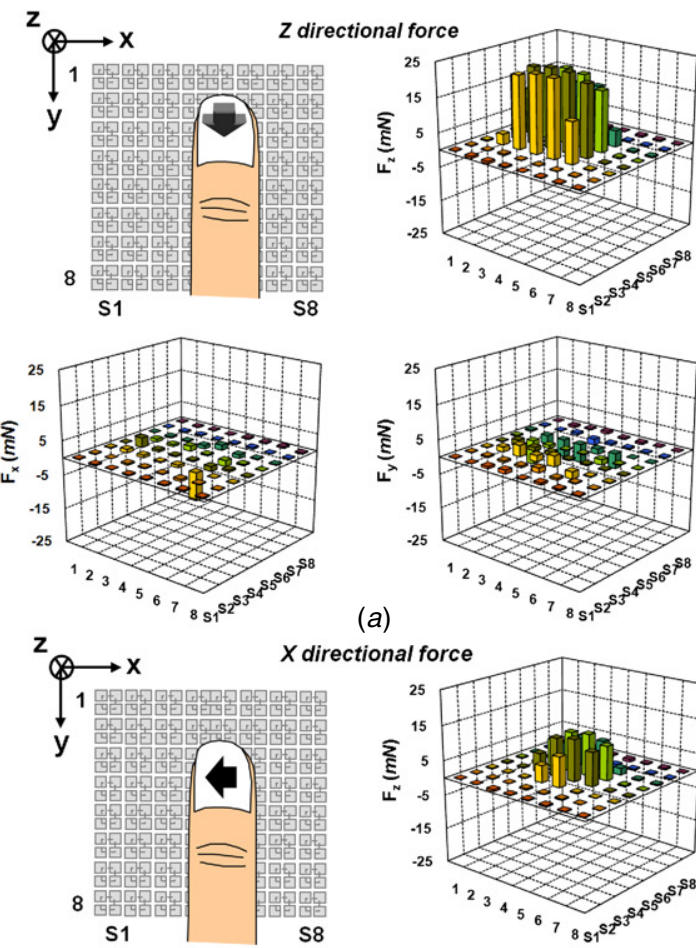

tional force
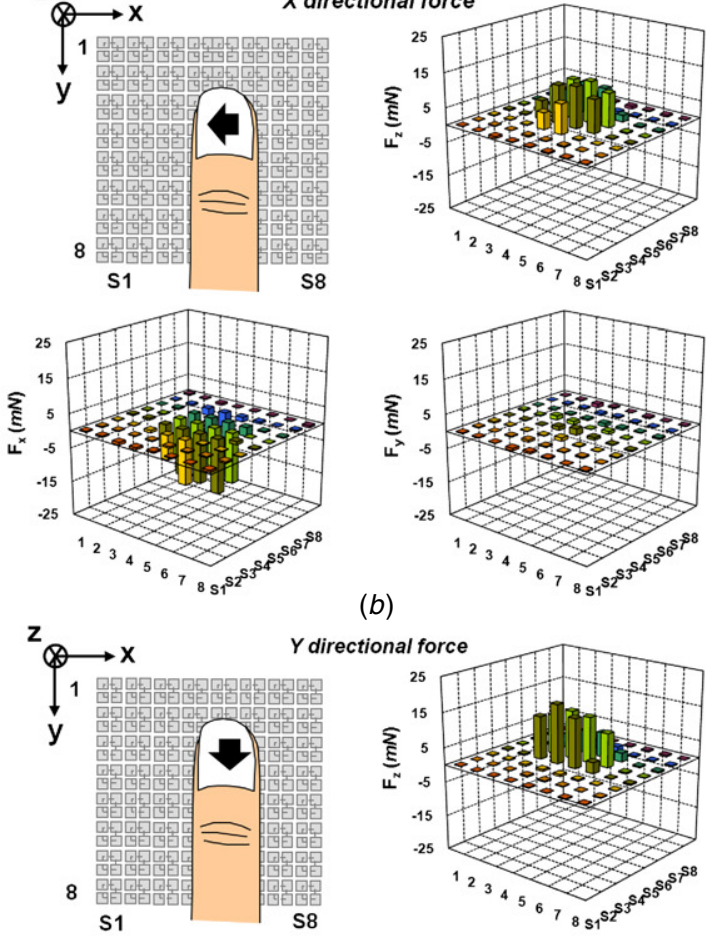

(b)
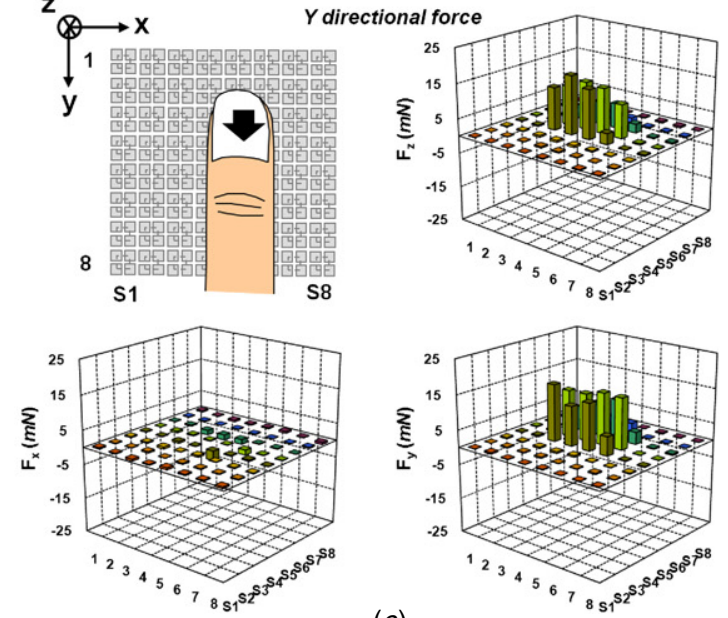

(c)

Figure 10. Snapshots of the measured axial components $\left(F_{x}, F_{y}\right.$ and $F_{z}$ ) of the contact force applied by a finger in each $(a) z_{-},(b) x-$ and $(c) y$-direction, respectively. The axial force components were calculated based on the model depicted in figure 7.

in the $z$-axis, $F_{z}$ is directly exerted on the spring element as shown in the figure. $F_{z}$ can also be derived using the REGD as

$$
F_{z}=k_{z} d_{z}=k_{z}\left[\frac{\Delta d_{\mathrm{eff} 12}+\Delta d_{\mathrm{eff} 22}+\Delta d_{\mathrm{eff} 11}+\Delta d_{\mathrm{eff} 21}}{4}\right] .
$$




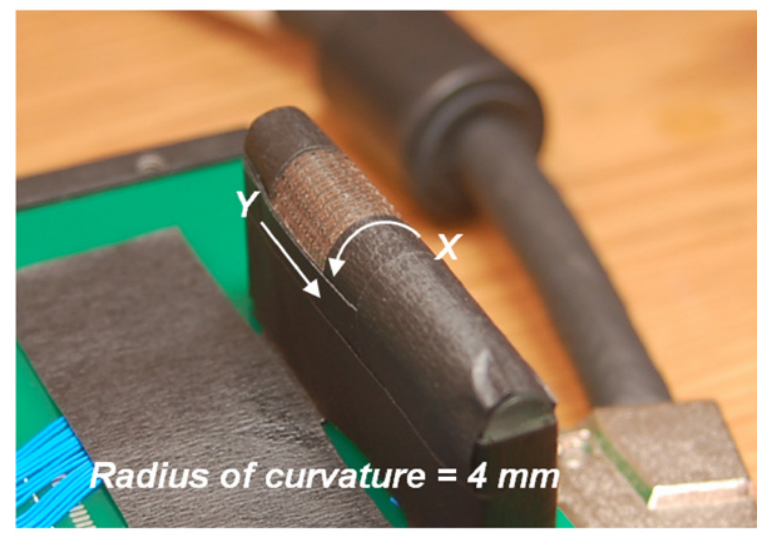

(a)

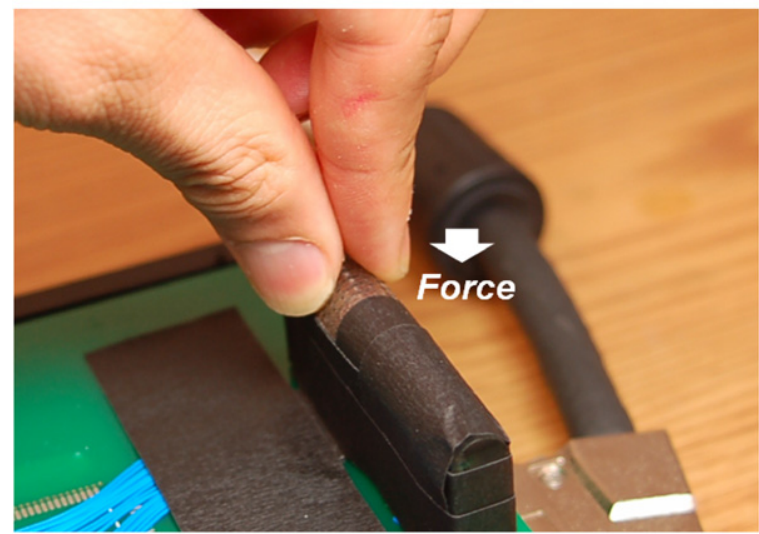

(b)

Figure 11. (a) Photograph of the fabricated three-axis tactile sensor attached on a cylindrical surface with $4 \mathrm{~mm}$ radius of curvature. $(b)$ Two fingers are applying a force on the sensor.

In equations (7)-(9), $d_{x}, d_{y}$ and $d_{z}$ are the displacements generated by the forces in each direction, and $k_{x}, k_{y}$ and $k_{z}$ are the corresponding spring constants.

The three axial displacements for the $x, y$, and $z$ axial forces from 0 to $15 \mathrm{mN}$ were calculated and are displayed in figure 8 . The displacements were calculated from the measured REGDs depicted in equations (7)-(9). The normal force shows only the $z$ displacement in figure $8(c)$. The shear force exhibits the displacements in only the corresponding axis while some in $z$ displacements due to the measurement setup mentioned above. They all show good linear responses. Our model seems to work fine in the $15 \mathrm{mN}$ force range. The spring constants for the $x$-, $y$ - and $z$-axes have been extracted from figure 8 as $9.62 \times 10^{3}, 1.04 \times 10^{4}$ and $1.04 \times 10^{4} \mathrm{~N}$ $\mathrm{m}^{-1}$, respectively. The estimated sensitivities of a unit sensor with a current setup were $1.3,1.2$ and $1.2 \% / \mathrm{mN}$ for the $x$-, $y$ and $z$-axes, respectively.

It can be expected that the fabricated sensor may show creep response when it is stressed because it is based on a viscoelastic material. However, we could not observe any creep response from the sensor while we were applying stress with the tip. We think this is because the minimum resolution of the force gauge $(1 \mathrm{mN})$ was not enough to observe subtle changes in the applied force.

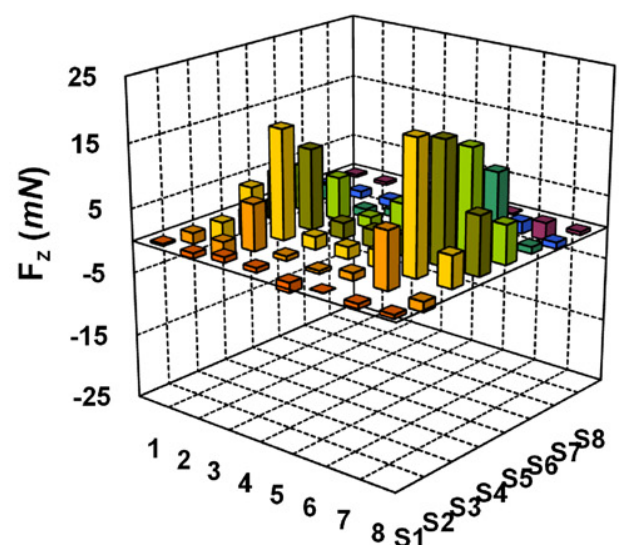

(a)

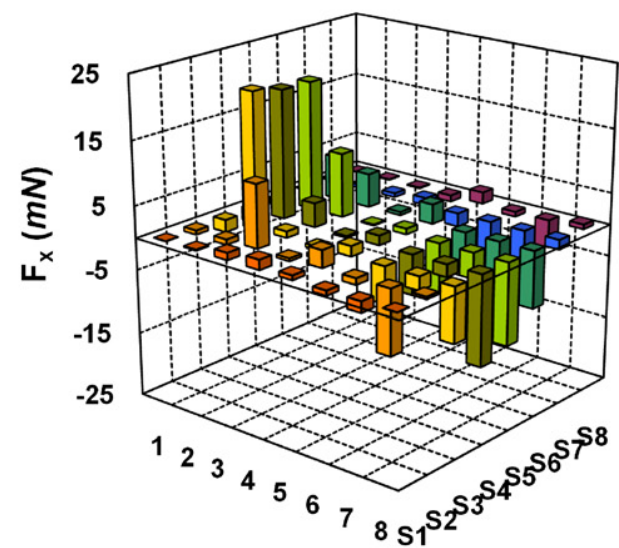

(b)

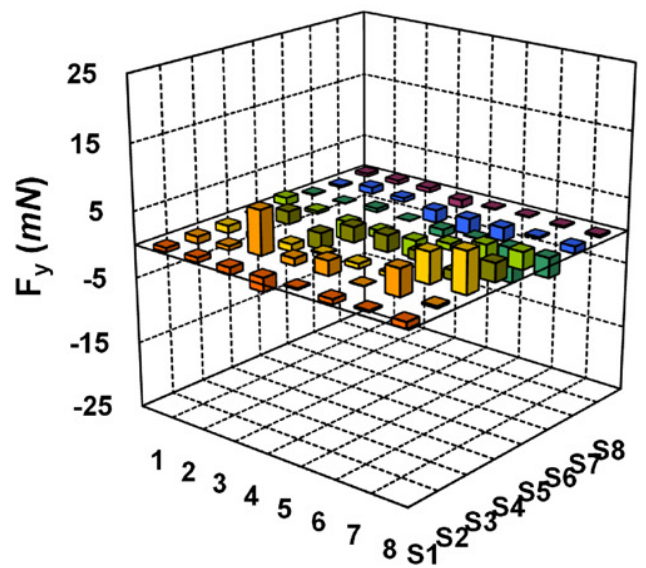

(c)

Figure 12. A snapshot of the measured axial components $\left(F_{x}, F_{y}\right.$ and $F_{z}$ ) of the contact force applied to the sensor attached on a curved surface shown in figure 11 .

\subsection{Real-time measurement of the contact force distribution in an array}

We built a custom measurement system to obtain real-time images of the contact force distribution in the three axes. A custom circuit board with analog switches and charge amplifiers, National Instruments' data acquisition pad and LabVIEW software have been used for this measurement system. The force components of all the sensors in an array are calculated in sequence and displayed in three different sections 
shown in figure 9 in real time. The display is divided into four sections. The upper left section displays the raw capacitance data from the capacitor array. The rest of the sections display the calculated axial components of the contact force.

The plots in figure 10 show snapshots of the measured distribution of contact forces applied by a human finger. Figure 10(a) displays the force components when a normal force ( $z$-direction) is applied. Only the $F_{z}$ component shows up clearly on the plots while there is no significant response in the shear components. For shear forces applied in the $x$ - and $y$ directions, only the corresponding shear force components, $F_{x}$ and $F_{y}$, appear as shown in figures $10(b)$ and $(c)$, respectively. However, there is also a response in the $z$-direction in both cases. This is because some normal force is required for enough friction to apply shear forces to the sensor. The frame rate of the current system is 6 frames per second. The frame rate limit came from the circuit board. It is not optimized for the fabricated sensor because it is our common test platform for various sensors. Higher frame rates (over 30) would have been obtained easily if a dedicated circuit board had been designed.

The dynamic response of the fabricated sensor was estimated to be faster than $160 \mathrm{~ms}$ because we could not observe any afterimage during the operation of the sensor.

Finally, we tested the flexibility of the fabricated sensor by attaching it to a half-cylindrical surface as shown in figure 11. The radius of curvature was $4 \mathrm{~mm}$ which is smaller than a human finger. Force distribution images were taken while two fingers were contacting the surface as shown in figure 11(b). The resultant force distributions are plotted in figure 12. The sensor operates without any flaws though a more sophisticated measurement is needed for a quantitative comparison. Since two fingers exerted shear force in opposite directions, $F_{x}$ shows two polarities at the same time in the figure.

\section{Conclusions}

In this work, we have successfully demonstrated the realtime measurement of the three-axis contact force distribution using a new three-axis capacitive tactile sensing array based on PDMS. The sensor can decompose the contact force into its normal and shear components. A unit sensor has four sensing capacitors separated by a wall structure. An $8 \times 8$ sensor array has successfully been fabricated and tested.

The fabricated sensor shows a balanced full-scale range of around $20 \mathrm{mN}$ in all three directions. A simplified model has been established to calculate the force components in each axis from the measured capacitance. The spring constants for the $x$-, $y$ - and $z$-axes were estimated to be $9.62 \times 10^{3}, 1.04 \times 10^{4}$ and $1.04 \times 10^{4} \mathrm{~N} \mathrm{~m}^{-1}$, respectively, from the measurement results and the model. The estimated sensitivities of a unit sensor for the current measurement setup were 1.3, 1.2 and $1.2 \% / \mathrm{mN}$ in the $x$-, $y$ - and $z$-directions, respectively.

The contact force distribution in all three axes was successfully visualized in real time for various applied forces. The mechanical response time of the sensor has been estimated to be less than $160 \mathrm{~ms}$. The fabricated sensor also showed decent responses on a curved surface. The remaining works will be the rigorous characterization of dynamic and creep responses and quantitative measurement of responses from the curved sensor by establishing a more sophisticated mechanical measurement setup.

By modifying the unit sensor structure, we have improved the unit sensor yield from $50 \%$ to $100 \%$ and the response time from more than $1 \mathrm{~s}$ to less than $160 \mathrm{~ms}$ compared to our previous sensor structure at the expense of some sensitivity.

The three-axis tactile sensor technology developed in this work is one of the promising technologies which can be applied not only to robotic or prosthetic hands for dexterously manipulating objects but also to computers and mobile devices as one of their intuitive touch interfaces.

\section{References}

[1] http://world.honda.com/ASIMO

[2] http://hubolab.kaist.ac.kr/

[3] Sally A 2009 The revolution will be prosthetized IEEE Spectr. 46 45-8

[4] http://www.suretouch.org/

[5] Puangmali P, Althoefer K, Senevirante L D, Murphy D and Dasgupta P 2008 State-of-the-art in force and tactile sensing for minimally invasive surgery IEEE Sensors $J$. 8 371-81

[6] Lee H-K, Chang S-I and Yoon E 2006 A flexible polymer tactile sensor: fabrication and modular expandability for large area deployment J. Microelectromech. Syst. 15 1681-6

[7] Beccai L, Roccella S, Ascari L, Valdastri P, Sieber A, Carroza M C and Dario P 2008 Development and experimental analysis of a soft compliant tactile microsensor for anthropomorphic artificial hand IEEE/ASME Trans. Mechatronics 13 158-68

[8] Zhu F and Spronck J W 1992 A capacitive tactile sensor for shear and normal force measurements Sensors Actuators A 31 115-20

[9] Cranny A, Cotton D P J, Chappell P H, Beeby S P and White N M 2005 Thick-film force and slip sensors for a prosthetic hand Sensors Actuators A 123-124 152-71

[10] Cotton D P J, Chappell P H, Cranny A, White N M and Beeby S P 2007 A novel thick-film piezoelectric slip sensor for a prosthetic hand IEEE Sensors $J$. 7 752-61

[11] Chu Z, Sarro P M and Middlehoek S 1996 Silicon three-axial tactile sensor Sensors Actuators A 54 505-10

[12] Kane B J, Cutkosky M R and Kovacs G T A 2000 A traction stress sensor array for use in high-resolution robotic tactile imaging J. Microelectromech. Syst. 9 425-33

[13] Kim K et al 2006 3-axes flexible tactile sensor fabricated by $\mathrm{Si}$ micromachining and packaging technology Proc. 19th IEEE Int. MEMS Conf. (Istanbul, Turkey, 22-26 January 2006) pp 678-81

[14] Hwang E-S, Seo J-H and Kim Y-J 2006 A polymer-based flexible tactile sensor for normal and shear load detection Proc. 19th IEEE Int. MEMS Conf. (Istanbul, Turkey, 22-26 January 2006) pp 714-7

[15] Engel J, Chen J and Liu C 2003 Development of polyimide flexible tactile sensor skin J. Micromech. Microeng. 13 359-66

[16] Lee H-K, Chung J, Chang S-I and Yoon E 2008 Normal and shear force measurement using a flexible polymer tactile sensor with embedded multiple capacitors J. Microelectromech. Syst. 17 934-42 